\title{
Protocol Chair at an Organization
}

National Cancer Institute

\section{Source}

National Cancer Institute. Protocol Chair at an Organization. NCI Thesaurus. Code C51855.

A person at a business or administrative group that retains the responsibility for regulation compliance and ensures the proper administration of the objectives, study design, and methodology of study. 